\title{
TRAVELLING WAVES FOR AN INFINITE LATTICE: MULTIBUMP TYPE SOLUTIONS
}

\author{
DiDiER SMETS
}

\section{Introduction}

We consider a one dimensional infinite lattice of particles with nearest neighbour interaction

$$
\ddot{q}_{k}(t)=\bar{V}_{x}\left(t, q_{k+1}(t)-q_{k}(t)\right)-\bar{V}_{x}\left(t, q_{k}(t)-q_{k-1}(t)\right), \quad k \in \mathbb{Z} .
$$

A solitary wave is a solution of (1) of the form

$$
q_{k}(t)=u(k-c t), \quad k \in \mathbb{Z} .
$$

Substituting in (1), we obtain the conditions

$$
\begin{aligned}
c^{2} u^{\prime \prime}(k-c t)= & \bar{V}_{x}(t, u(k+1-c t)-u(k-c t)) \\
& -\bar{V}_{x}(t, u(k-c t)-u(k-1-c t)), \quad k \in \mathbb{Z}, t \in \mathbb{R} .
\end{aligned}
$$

Assuming further that $\bar{V}(\cdot, x)$ is $1 / c$-periodic for each $x \in \mathbb{R}$, equations (2) reduce to the second order forward-backward differential-difference equation:

$$
c^{2} u^{\prime \prime}(t)=\bar{V}_{x}(-t / c, u(t+1)-u(t))-\bar{V}_{x}(-t / c, u(t)-u(t-1)) .
$$

1991 Mathematics Subject Classification. Primary: 58E05; Secondary: 34C25.

Key words and phrases. Lattice equations, multibump solutions, mini-max theorems, travelling waves.

Works supported by the FNRS, Belgium. 
In a preceding work (see [9]), we studied the autonomous case corresponding to $\bar{V}(t, x)=V(x)$. Under the assumptions:

$$
\begin{cases}V(x)=\lambda \frac{x^{2}}{2}+W(x), & W \in \mathcal{C}^{1}(\mathbb{R}, \mathbb{R}), \\ W(0)=0, W^{\prime}(x)=o(|x|), & x \rightarrow 0,\end{cases}
$$

and

$$
\left\{\begin{array}{l}
\sup _{\mathbb{R}} W>0 \text { and there exists } \alpha>2 \text { such that } \\
\text { for all } \quad x \in \mathbb{R}, \alpha W(x) \leq x W^{\prime}(x)
\end{array}\right.
$$

we proved:

TheOREM 1.1. For every $c$ such that $c^{2}>\max (0, \lambda)$, equation (3) has a nontrivial classical solution $u$ such that $u^{\prime} \in L^{2}(\mathbb{R})$.

This theorem extends without any change in the setting that will be our. The solutions were found as critical points of the functional

$$
\varphi(u):=\int_{\mathbb{R}}\left[\frac{c^{2}}{2}\left(u^{\prime}(t)\right)^{2}-V(u(t+1)-u(t))\right] d t
$$

defined on the space

$$
X:=\left\{u \in H_{\mathrm{loc}}^{1}(\mathbb{R}): u^{\prime} \in L^{2}(\mathbb{R}), u(0)=0\right\} .
$$

To overcome the non-compactness due to "translation" invariance, we used a weak convergence argument together with Lieb's lemma. These arguments are the first steps to establish the caracterisation of Palais-Smale sequences for $\varphi$.

In this work, we will prove a multiplicity result in the non-autonomous case. More precisely, we will search for infinitely many solutions of (3) of multibump type.

The methods we use are originated in the works of Coti Zelati, Ekeland, Séré (see [3], [7]) and after refined by Rabinowitz, Coti Zelati ([4], [5]), Arioli, Caldiroli, Gazzola, Montecchiari and Terracini ([1], [2]).

In all these works, the functionals are invariant under a non compact but discrete group (or the product of such a group with a compact one). In the autonomous case, our problem is invariant under a group isomorphic to $\mathbb{R}$ for which the above methods don't seem to work.

Moreover, in the special case of the integrable Toda lattice, it is known from scattering theory that solitons are found in relation with bound states of a linear spectral problem, those of which are of finite number. Of course, solitary waves are not necessarily solitons but this argument shows it seems difficult to find multibump solutions in the general autonomous setting.

From the physical point of view, the assumption of time periodic dependence for the potential is not without any sense. Many phenomenons related to a lattice are affected by periodic changes such as luminosity, temperature or others. 
Acknowledgement. I am indebted to my thesis advisor, Professor Michel Willem, for introducing me to this problem. I also acknowledge him for his constant help and encouragement.

\section{Functional seting and some previous results}

From now on, we will asume that

$$
\bar{V}(t, x):=p(t) V(x),
$$

with $V$ satisfying $\left(\mathrm{V}_{1}\right),\left(\mathrm{V}_{2}\right)$ and $p \in \mathcal{C}(\mathbb{R}, \mathbb{R})$ is positive, $1 / c$-periodic and $|p|_{\infty}=1$.

For simplicity, we will denote $P(t):=p(-t / c)$, thus obtaining a 1-periodic function. We also define the linear operator $A$ by $A u(t):=u(t+1)-u(t)$, and the energy functional by:

$$
\varphi(u):=\int_{\mathbb{R}}\left[\frac{c^{2}}{2}\left(u^{\prime}\right)^{2}-P \cdot V(A u)\right]
$$

on the space

$$
X:=\left\{u \in H_{\mathrm{loc}}^{1}(\mathbb{R}): u^{\prime} \in L^{2}(\mathbb{R}), u(0)=0\right\},
$$

endowed with the norm $\|u\|:=\left|u^{\prime}\right|_{2}$.

Notations. For a set $A$ and a positive number $\delta, A_{\delta}$ will denote the points whose distance to $A$ is less or equal than $\delta$. When $A$ is finite or at least discrete, we will also use $\mathbf{B}(A, \delta)$ to represent the same set. For a real functional $\varphi, \varphi^{d}$ will denote the points $x$ in the domain of $\varphi$ such that $\varphi(x) \leq d$. Finally, $\operatorname{supp}(u)$ denotes the support of the function $u$.

The following results have been proved in [8], [9] or are trivial adaptations of some therein.

Lemma 2.1. Any critical point $u$ of $\varphi$ is a classical solution of (3).

Lemma 2.2. The functional $\varphi$ belongs to $\mathcal{C}^{1}(X, \mathbb{R})$ and satisfies the assumptions of the Mountain Pass Lemma, i.e. there exist $r>0, e \in X$ with $\|e\|>r$ and

$$
b:=\inf _{\|u\|=r} \varphi(u)>\varphi(0) \geq \varphi(e) .
$$

The corresponding inf max value will be denoted by $d$.

Lemma 2.3. If $\left(u_{n}\right) \subset X$ is a Palais-Smale sequence for $\varphi$ at level $l$ then $l \geq 0$.

Lemma 2.4. There exists $C_{1}>0$ such that any non trivial critical point $u$ of $\varphi$ satisfies $\varphi(u) \geq C_{1}$. 
LEMma 2.5. There exists $C_{2}>0$ such that any non trivial critical point $u$ of $\varphi$ satisfies $\|u\| \geq C_{2}$.

For a function $u \in X$, we define

$$
\tau_{a} u(t):=u(t-a)-u(-a) .
$$

It is clear from the definition that $\varphi$ is invariant under the group $\left(\tau_{k}\right)_{k \in \mathbb{Z}}$. The following lemma characterises the non-compactness of the Palais-Smale sequences.

Lemma 2.6. Let $\left(u_{n}\right) \subset X$ a Palais-Smale sequence for $\varphi$ at level $l$. Then there exist a subsequence (still denoted by $u_{n}$ ), an integer $k \leq l / C_{1}-l$, $k$ sequences $\left(y_{n}^{i}\right)_{1 \leq i \leq k} \subset \mathbb{Z}^{k}$ and $k+1$ critical points $v_{0}, \ldots, v_{k}$ of $\varphi$ such that
(a) $\left|y_{n}^{i}\right| \rightarrow \infty$ for all $i$,
(b) $\left|y_{n}^{i}-y_{n}^{j}\right| \rightarrow \infty$ for all $i \neq j$,
(c) $l=\sum_{i=0}^{k} \varphi\left(v_{i}\right)$,
(d) $\left\|u_{n}-v_{0}-\sum_{i=1}^{k} \tau_{y_{n}^{i}} v_{i}\right\| \rightarrow 0$.

REMARK 2.7. In [8], Lemma 2.6 was proved under the supplementary assumption that $V \in \mathcal{C}^{2}$.

\section{Multibump solutions}

Following Coti Zelati, Ekeland and Séré [3], we will assume a "non-degeneracy" condition for $\varphi$, more precisely:

$$
\text { there exists } \varepsilon_{0}>0 \text { such that } \mathcal{F} / \mathbb{Z} \text { is finite, }
$$

where $\mathcal{F}:=\left\{u \in X \backslash\{0\}: \varphi^{\prime}(u)=0, \varphi(u)<d+\varepsilon_{0}\right\}$ and the quotient is understood in connection with the invariance under the $\left(\tau_{k}\right)_{k \in \mathbb{Z}}$ group.

REMARK 3.1. Notice that if condition $(*)$ fails, then by Lemma 2.1, equation (3) admits infinitely many solutions up to symmetries.

We pick up one element in each equivalence class of $\mathcal{F} / \mathbb{Z}$ and call $F$ the resulting set. Following Caldiroli and Montecchiari [2], we will say that a critical point $u$ is a multibump solution of kind $(n, \rho)$ if there exists $k_{1}, \ldots, k_{n} \in \mathbb{Z}$ such that

$$
u \in B\left(\sum_{i=1}^{n} \tau_{k_{i}} F, \rho\right) .
$$

Our main theorem writes

ThEOREM 3.2. Suppose $\left(\mathrm{V}_{1}\right)-\left(\mathrm{V}_{3}\right),(*)$ are satisfied and $\left(\mathrm{V}_{4}\right) \quad$ there exists $\varepsilon_{1}>0$ such that $V$ is concave in $\left[-\varepsilon_{1}, \varepsilon_{1}\right]$. 
Then for every $\rho>0, k_{1}<\ldots<k_{n} \in \mathbb{Z}$ there exist $N \in \mathbb{N}$ and a critical point $u$ of $\varphi$ such that

$$
u \in B\left(\sum_{i=1}^{n} \tau_{k_{i} N} F, \rho\right) .
$$

In particular, for every $\rho>0, n \in \mathbb{N}$, there exist infinitely many multibump solutions of kind $(n, \rho)$.

This theorem will be proved in the next section.

\section{Proof of the main result}

Henceforth, we assume that the assumptions of Theorem 3.2 are satisfied. We start this section by a lemma that somewhat says that non convergent PalaisSmale sequences are not too close to critical points.

Lemma 4.1. Let $G:=\left\{\sum_{i=1}^{l} \tau_{k_{i}} u_{i}: 1 \leq l \leq\left(d+\varepsilon_{0}\right) / C_{1}, k_{i} \in \mathbb{Z}, u_{i} \in F\right\}$. Then we have $0<\varepsilon_{2}:=\inf _{\substack{u, v \in G \\ u \neq v}}\|u-v\|$.

PROOF. If not, there exist sequences $k_{i}^{n}, h_{i}^{n}, u_{i}^{n}$ and $v_{i}^{n}$ such that:

$$
0 \neq\left\|\sum_{i=1}^{l_{1}} \tau_{k_{i}^{n}} u_{i}^{n}-\sum_{i=1}^{l_{2}} \tau_{h_{i}^{n}} v_{i}^{n}\right\| \rightarrow 0 .
$$

By invariance, we can suppose that $k_{1}^{n}=0$. Moreover, we can assume going if necessary to a subsequence that

(1) $u_{i}^{n}=u_{i}^{m}=u_{i}$ for all $m, n \in \mathbb{N}$,

(2) $k i^{n}=k_{i}^{m}=k_{i}$ if $\left(k_{i}^{j}\right)_{j \in \mathbb{N}}$ is bounded,

(3) $k_{i}^{n} \rightarrow \infty$ if $\left(k_{i}^{j}\right)_{j \in \mathbb{N}}$ is unbounded,

and the same for $v_{i}^{n}$ and $h_{i}^{n}$. Thus we have,

$$
\begin{aligned}
0 & =\lim _{n \rightarrow \infty}\left\|\sum_{i=1}^{l_{1}} \tau_{k_{i}^{n}} u_{i}^{n}-\sum_{i=1}^{l_{2}} \tau_{h_{i}^{n}} v_{i}^{n}\right\|^{2} \\
& =\left\|\sum \tau_{k_{i}} u_{i}-\sum \tau_{h_{i}} v_{i}\right\|^{2}+\lim _{n \rightarrow \infty}\left\|\sum \tau_{k_{i}^{n}} u_{i}-\sum \tau_{h_{i}^{n}} v_{i}\right\|^{2}
\end{aligned}
$$

so that

$$
\left\{\begin{array}{l}
\sum \tau_{k_{i}} u_{i}=\sum \tau_{h_{i}} v_{i}, \\
0 \neq \mid \sum \tau_{k_{i}^{n}} u_{i}-\sum \tau_{h_{i}^{n}} v_{i} \| \rightarrow 0 .
\end{array}\right.
$$

At this level, we repeat the preceding operations, that is translating by invariance and going to a subsequence. After at most $2\left(d+\varepsilon_{0}\right) / C_{1}$ cycles, we will get the contradiction:

$$
\sum_{i=1}^{l_{1}} \tau_{k_{i}} u_{i}=\sum_{i=1}^{l_{2}} \tau_{h_{i}} v_{i}
$$

whereas the initial sequence was made of non-zero elements only. 
Let $\rho$ and $k_{1}<\ldots<k_{n}$ as given in Theorem 3.2. As $F$ is finite, there exists $C_{3}>0$ such that $\mathbf{B}\left(F, C_{3}\right) \subset \varphi^{-1}\left[0, d+\varepsilon_{0}\right.$ [. Without loss of generality, we can assume $\rho<\min \left(\varepsilon_{1}, \varepsilon_{2}, C_{2}, C_{3}\right) / 2$. Define $\delta:=\rho / 15 n$.

Lemma 4.2. There exists $0<\varepsilon<\varepsilon_{0} / 2$ such that

$$
\text { for every } u \in \varphi^{-1}\left[0, d+\varepsilon_{0}\left[\cap\left(X \backslash G_{\delta}\right), \quad\left\|\varphi^{\prime}(u)\right\|>8 \varepsilon / \delta .\right.\right.
$$

Proof. If not, there would exist a Palais-Smale sequence $\left(u_{n}\right) \subset X \backslash G_{\delta} \cap$ $\varphi^{-1}\left[0, d+\varepsilon_{0}\left[\right.\right.$. But by Lemmas 2.6 and $2.4\left(u_{n}\right) \rightarrow G$, a contradiction.

Lemma 4.3. There exist $\gamma:[0,1] \longrightarrow F_{5 \delta}$ and $R_{1}>0$ verifying:

(a) $\varphi(\gamma(0)) \leq d-\varepsilon, \varphi(\gamma(1)) \leq d-\varepsilon$,

(b) $\max _{t \in[0,1]} \varphi(\gamma(t)) \leq d+\varepsilon / n$,

(c) $\operatorname{supp}[\gamma(t)]^{\prime} \subset\left[-R_{1}, R_{1}\right]$ for every $t \in[0,1]$.

(d) $\inf _{\widetilde{\gamma} \in \Gamma} \max _{t \in[0,1]} \varphi(\widetilde{\gamma}(t)) \geq d$,

where $\Gamma:=\{\widetilde{\gamma}:[0,1] \rightarrow X, \widetilde{\gamma}(0)=\gamma(0), \widetilde{\gamma}(1)=\gamma(1)\}$.

Proof. Let $S:=X \backslash \mathcal{F}_{3 \delta}$. As $\rho<\varepsilon_{2} / 2$, one has $S_{\delta} \backslash S=\mathcal{F}_{3 \delta} \backslash \mathcal{F}_{2 \delta} \subset X \backslash G_{2 \delta}$, Lemma 4.2 gives

$$
\left\|\varphi^{\prime}(u)\right\| \geq 8 \varepsilon / \delta, \quad \text { for all } u \in \varphi^{-1}[d-\varepsilon, d+\varepsilon] \cap\left(S_{\delta} \backslash S\right) .
$$

By Lemmas 2.6 and 4.1, any Palais-Smale sequence at a level $b \in[d-2 \varepsilon, d+2 \varepsilon]$ such that $\left\|u_{n+1}-u_{n}\right\| \rightarrow 0$ contains a subsequence converging to an element $u \in \mathcal{F}$. Besides, $S_{2 \delta} \cap \mathcal{F}=\emptyset$.

Let $\eta$ the deformation given by Lemma 4.9 and $\gamma_{0}:[0,1] \rightarrow X$ such that

(1) $\gamma_{0}(0)=0, \gamma_{0}(1)=e$,

(2) $\max _{t \in[0,1]} \gamma_{0}(t) \leq d+\varepsilon / n$.

We will denote $\gamma_{1}$ the deformed path, i.e. $\gamma_{1}(t)=\eta\left(1, \gamma_{0}(t)\right)$. There exists $t \in[0,1]$ with $\gamma_{0}(t) \in F_{3 \delta}$ and $\varphi\left(\gamma_{0}(t)\right) \geq d$. Indeed, otherwise we would have $\max _{t \in[0,1]} \gamma_{1}(t)<d$, a contradiction.

Let $A_{1}, \ldots, A_{m}$ be the largest intervals in $[0,1]$ satisfying $\gamma_{0}\left(A_{i}\right) \subset \mathcal{F}_{3 \delta}$ for each $i$. There is at least one $i \in\{1, \ldots, m\}$, say $i_{0}$, such that:

$$
\inf _{\widetilde{\gamma}_{1} \in \Gamma^{i}} \max _{t \in A_{i}} \varphi\left(\widetilde{\gamma}_{1}(t)\right) \geq d,
$$

where $\Gamma^{i}:=\left\{\widetilde{\gamma}_{1}: A_{i} \rightarrow X, \widetilde{\gamma}_{1}=\gamma_{1}\right.$ on $\left.\partial A_{i}\right\}$.

Let $l \in \mathbb{N}$ such that $\tau_{l} \gamma_{0}\left(A_{i_{0}}\right) \subset F_{3 \delta}$, define $\gamma_{2}:[0,1] \rightarrow X$ so that $\gamma_{2}([0,1])=$ $\tau_{l} \gamma_{1}\left(A_{i_{0}}\right)$. As $F_{4 \delta} \backslash F_{3} \delta \subset X \backslash G_{\delta}$, it is clear that $\gamma_{2}([0,1]) \subset F_{4 \delta}$. By construction and Lemma 4.9, $\gamma_{2}$ satisfies properties (a), (b) and (d). By compactness, we can choose $R_{1}$ sufficiently large such that if

$$
[\gamma(t)]^{\prime}(x):=\left[\gamma_{2}(t)\right]^{\prime}(x) \cdot \chi_{\left[-R_{1}, R_{1}\right]}(x),
$$


then $\gamma$ satisfies $(\mathrm{a})-(\mathrm{d})$ and $\gamma([0,1]) \subset F_{5 \delta}$.

Suppose that $N$ is fixed and define, for $1 \leq i \leq n-1$,

$$
I_{j}:=\left[N \cdot k_{j}+N / 3, N \cdot k_{j+1}-N / 3\right] \text {. }
$$

We will note $M B:=\mathbf{B}\left(\sum_{i=1}^{n} \tau_{k_{i} N} F, \rho\right)$.

LEMMA 4.4. If $N$ is sufficiently large, there exists a continuous "dissociating" function $\mathbf{D}: M B \rightarrow(X)^{n}$ such that if we note $\mathbf{D}(u) \equiv\left(u^{1}, \ldots, u^{n}\right)$ then for all $u \in M B$

$$
\left\{\begin{array}{l}
u=\sum_{i=1}^{n} u^{i} \\
\varphi(u) \geq \sum_{i=1}^{n} \varphi\left(u^{i}\right)-\varepsilon / 2 n .
\end{array}\right.
$$

Moreover, if $u=\sum_{i=1}^{n} w_{i}$ with $\operatorname{supp}\left[w_{i}\right]^{\prime} \subset\left[k_{i}-N / 3, k_{i}+N / 3\right]$ then $u^{i}=w_{i}$ for each $i$.

Proof. Fix $j \in\{1, \ldots, n-1\}$. As $F$ is finite, for $N$ large enough we have:

$$
\left[\int_{\mathbb{R} \backslash[-N / 3, N / 3]}\left(u^{\prime}\right)^{2}\right]^{1 / 2}<\frac{\rho}{n} \text { for all } u \in F .
$$

Thus, for every $u \in M B$, there exist $t_{j}^{u} \in I_{j} \cap \mathbb{Z}$ and an open neighbourhood $\mathcal{O}_{j}^{u} \subset X$ of $u$ with:

$$
\left[\int_{t_{j}^{u}-1}^{t_{j}^{u}+1}\left|\omega^{\prime}\right|^{2}\right]^{1 / 2}<\frac{12 \rho}{N} \quad \text { for all } \omega \in \mathcal{O}_{j}^{u}
$$

The familly $\left\{\mathcal{O}_{j}^{u}, u \in M B\right\}$ forms an opening covering of $M B$. Let $\left\{\mathcal{O}_{j}^{v}, v \in V_{j}\right\}$ a locally finite covering of $M B$ finer than the previous one and $\left\{p_{j}^{v}, v \in V_{j}\right\}$ a continuous partition of unity associated to it. We define, for $2 \leq j \leq n-1$,

$$
\left(u^{j}\right)^{\prime}(t):=u^{\prime}(t) \cdot\left\{\sum_{V_{j}} p_{j}^{v}(u) \chi_{]_{-\infty, t_{j}^{v}}}(t)\right\} \cdot\left\{\sum_{V_{j-1}} p_{j-1}^{v}(u) \chi_{\left[t_{j-1}^{v}, \infty[\right.}(t)\right\}
$$

and

$$
\begin{aligned}
& \left(u^{1}\right)^{\prime}(t):=u^{\prime}(t) \cdot\left\{\sum_{V_{1}} p_{1}^{v}(u) \chi_{]-\infty, t_{1}^{v}[}(t)\right\}, \\
& \left(u^{n}\right)^{\prime}(t):=u^{\prime}(t) \cdot\left\{\sum_{V_{n-1}} p_{n-1}^{v}(u) \chi_{\left[t_{n-1}^{v},+\infty[\right.}(t)\right\} .
\end{aligned}
$$

Clearly, $u=\sum_{i=1}^{n} u^{i}$ and the continuity of $\mathbf{D}$ is a consequence of the one of the $\left(p_{j}^{v}\right)$. It remains to prove that $\varphi(u) \geq \sum_{i=1}^{n} \varphi\left(u^{i}\right)-\varepsilon / 2 n$. For $u \in M B$, denote $V_{j}^{u}:=\left\{v \in V_{j}\right.$ s.t. $\left.p_{j}^{v}(u) \neq 0\right\}$.

(a) As $u^{\prime},\left(u^{j}\right)^{\prime}$ have equal signs, $\left|u^{\prime}(t)\right|^{2} \geq \sum_{j}\left|\left(u^{j}\right)^{\prime}(t)\right|^{2}$ and thus,

$$
\int_{\mathbb{R}} \frac{c^{2}}{2}\left(u^{\prime}\right)^{2} \geq \sum_{j} \int_{\mathbb{R}} \frac{c^{2}}{2}\left(\left(u^{j}\right)^{\prime}\right)^{2}
$$


(b) If $t \in I_{j} \backslash \bigcup_{V_{j}^{u}}\left[t_{j}^{v}-1, t_{j}^{v}\right]$,

(5) $\quad V\left(A u^{j}(t)\right)=V\left(\sum_{V_{j}^{u}} p_{j}^{v} \int_{t}^{t+1} u^{\prime}(s) \chi_{-\infty, t_{j}^{v}}(s) d s\right)$

$$
\geq \sum_{V_{j}^{u}} p_{j}^{v} V\left(\int_{t}^{t+1} u^{\prime}(s) \chi_{-\infty, t_{j}^{v}[}(s) d s\right)=\sum_{\substack{V_{j}^{u} \\ t_{j}^{v} \geq t}} p_{j}^{v} V(A u(t)),
$$

where we used $\rho<\varepsilon_{1}$ and the concavity of $V$ in $\left[-\varepsilon_{1}, \varepsilon_{1}\right]$.

Similarly, we obtain

$$
V\left(A u^{j+1}(t)\right) \geq \sum_{\substack{V_{j}^{u} \\ t_{j}^{v} \leq t}} p_{j}^{v} V(A u(t))
$$

and thus $V\left(A u^{j}(t)\right)+V\left(A u^{j+1}(t)\right) \geq \sum_{V_{j}^{u}} p_{j}^{v} V(A u(t))=V(A u(t))$. Observe also that, if $i \neq j$ or $j+1$, then $V\left(A u^{i}(t)\right)=V(0)=0$.

(c) If $t \in\left[t_{j}^{v}-1, t_{j}^{v}\right]$,

$$
\begin{aligned}
V & \left(A u^{j}(t)\right)+V\left(A u^{j+1}(t)\right)-V(A u(t)) \\
\geq & \sum_{V_{j}^{u}} p_{j}^{v} V\left(\int_{t}^{t+1} u^{\prime}(s) \chi_{-\infty, t_{j}^{v}}(s) d s\right) \\
& +\sum_{V_{j}^{u}} p_{j}^{v} V\left(\int_{t}^{t+1} u^{\prime}(s) \chi_{\left[t_{j}^{v},-\infty[\right.}(s) d s\right)-V\left(\int_{t}^{t+1} u^{\prime}(s) d s\right) \\
= & \sum_{t_{j}^{v} \geq t+1} p_{j}^{v} V(A u(t))+\sum_{t_{j}^{v} \in[t, t+1[} p_{j}^{v} V\left(\int_{t}^{t+1} u^{\prime}(s) \chi_{]-\infty, t_{j}^{v}[}(s) d s\right) \\
& +\sum_{t_{j}^{v} \leq t} p_{j}^{v} V(A u(t))+\sum_{t_{j}^{v} \in[t, t+1[} p_{j}^{v} V\left(\int_{t}^{t+1} u^{\prime}(s) \chi_{\left[t_{j}^{v}, \infty[\right.}(s) d s\right)-V(A u(t)) \\
= & \sum_{t_{j}^{v} \in[t, t+1[} p_{j}^{v}\left[V\left(\int_{t}^{t+1} u^{\prime}(s) \chi_{-\infty, t_{j}^{v}[}(s) d s\right)\right. \\
& \left.+V\left(\int_{t}^{t+1} u^{\prime}(s) \chi_{\left[t_{j}^{v},-\infty[\right.}(s) d s\right)\right]-\sum_{t_{j}^{v} \in[t, t+1[} p_{j}^{v} V(A u(t)) \\
\geq & 0-\sum_{t_{j}^{v} \in[t, t+1[} p_{j}^{v} \cdot 2 \cdot(|\lambda|+1) \cdot \int_{t}^{t+1}\left(u^{\prime}(s)\right)^{2} d s \\
\geq & -2(|\lambda|+1)\left(\frac{\rho}{15 N}\right)^{2}\left(\sum_{t_{j}^{v} \in[t, t+1[} p_{j}^{v}\right) .
\end{aligned}
$$


Integrating on $\left[t_{j}^{v}-1, t_{j}^{v}\right]$ and summing for $t_{j}^{v} \in V_{j}^{u}$ we get

$$
\begin{aligned}
\int_{\bigcup_{t_{j}^{v} \in V_{j}^{u}}^{u}\left[t_{j}^{v}-1, t_{j}^{v}\right]} P \cdot\left[V\left(A u^{j}\right)+V\left(A u^{j+1}\right)\right. & -V(A u)] \\
& \geq-2(|\lambda|+1)\left(\frac{\rho}{15 N}\right)^{2} .
\end{aligned}
$$

Then summing on $j$ an collecting the result with points (a) and (b)

$$
\varphi(u) \geq \sum_{j} \varphi\left(u^{j}\right)-2(n-1)(|\lambda|+1)\left(\frac{\rho}{15 N}\right)^{2} .
$$

It suffices then to take $N$ sufficiently large so that $2(n-1)(|\lambda|+1)(\rho / 15 N)^{2}<$ $\varepsilon / 2 n$.

Using similar arguments, we obtain

LEMmA 4.5. If $N$ is sufficiently large, there exists a "dissociating" function (no longer continuous) $\overline{\mathbf{D}}: M B \rightarrow(X)^{n}$ such that if we note $\overline{\mathbf{D}}(u) \equiv$ $\left(u_{1}, \ldots, u_{n}\right)$ then for all $u \in M B$

$$
\left\{\begin{array}{l}
u=\sum_{i} u_{i} \text { and }\|u\|=\sum_{i}\left\|u_{i}\right\|, \\
\left\|\varphi^{\prime}(u)\right\| \geq\left\|\varphi^{\prime}\left(u_{i}\right)\right\|-4 \varepsilon / \delta \quad 1 \leq i \leq n .
\end{array}\right.
$$

Moreover, if $u=\sum_{i} \tau_{k_{i} N} w_{i} \in \sum_{i} \tau_{k_{i} N} F$ then $\left\|u_{i}-\tau_{k_{i} N} w_{i}\right\| \leq \delta$ for each $1 \leq i \leq n$.

Henceforth, we will assume that $N$ has been chosen large enough so that Lemmas 4.4, 4.5 are valid and $R_{1}<N / 3$. We will prove that for this $N$, equation (3) admits a solution $u \in M B$. Define the $n$-surface

$$
\Psi:[0,1]^{n} \rightarrow \mathbf{B}\left(\sum_{i} \tau_{k_{i} N} F, \rho / 3\right) \quad\left(x_{1}, \ldots, x_{n}\right) \mapsto \sum_{i} \tau_{k_{i} N} \gamma\left(x_{i}\right)
$$

$\left(\gamma\right.$ is defined in (4.3)). Clearly, for every $x \in \partial[0,1]^{n}, \varphi(\Psi(x)) \leq(d-\varepsilon)+(d+$ $\varepsilon / n)+\ldots+(d+\varepsilon / n)=n d-\varepsilon / n$. Also define

$$
\Theta:=\left\{\bar{\Psi}:[0,1]^{n} \rightarrow M B \text { continuous with } \bar{\Psi}=\Psi \text { on } \partial[0,1]^{n}\right\}
$$

and

$$
\theta:=\inf _{\bar{\Psi} \in \Theta} \max _{x \in[0,1]^{n}} \varphi(\bar{\Psi}(x))
$$

LEMMA 4.6. $\theta \leq n d+\varepsilon$.

PROOF. $\max _{x \in[0,1]^{n}} \varphi(\Psi(x)) \leq n(d+\varepsilon / n)=n d+\varepsilon$. 
LEMMA 4.7. $\theta \geq n d-\varepsilon / 2 n$.

Proof. Let $\bar{\Psi} \in \Theta$ and $l_{i}:[0,1] \rightarrow[0,1]^{n}$ a path joining the two faces $\left\{x_{i}=0\right\}$ and $\left\{x_{i}=1\right\}$. Define $\overline{l_{i}}:[0,1] \rightarrow X$ by $\overline{l_{i}}(t):=\left[\bar{\Psi}\left(l_{i}(t)\right)\right]^{i}$. We have, $\overline{l_{i}}(0)=\tau_{k_{i} N} \gamma(0)$ and $\overline{l_{i}}(1)=\tau_{k_{i} N} \gamma(1)$. By Lemma 4.3 and invariance, $\max _{t \in[0,1]} \overline{l_{i}}(t) \geq d$. Hence, the sets $E_{i}:=\left\{x \in[0,1]\right.$ s.t. $\left.[\bar{\Psi}(x)]^{i} \geq d\right\}$ separate the two faces $\left\{x_{i}=0\right\}$ and $\left\{x_{i}=1\right\}$. By a theorem of Hurewicz [6], $\bigcap_{i} E_{i} \neq \emptyset$.

Now, if $x \in \bigcap_{i} E_{i}$, Lemma 4.4 gives

$$
\varphi(\bar{\Psi}(x)) \geq \sum_{i} \varphi\left([\bar{\Psi}(x)]^{i}\right)-\frac{\varepsilon}{2 n} \geq n d-\frac{\varepsilon}{2 n} .
$$

LEMma4.8. For every $u \in \mathbf{B}\left(\sum_{i} \tau_{k_{i} N} F, 2 \rho / 3\right) \backslash \mathbf{B}\left(\sum_{i} \tau_{k_{i} N} F, \rho / 3\right)$,

$$
\left\|\varphi^{\prime}(u)\right\| \geq 4 \varepsilon / \delta=60 n \varepsilon / \rho .
$$

Proof. Let $v=\sum_{i} \tau_{k_{i} N} w_{i} \in \sum_{i} \tau_{k_{i} N} F$ with $\|v-u\| \leq 2 \rho / 3+\delta$. As

$$
\frac{\rho}{3} \leq\|v-u\|=\left\|\sum_{i} v_{i}-u_{i}\right\|=\sum_{i}\left\|v_{i}-u_{i}\right\|,
$$

there is at least one $i \in\{1, \ldots, n\}$ verifying $\left\|v_{i}-u_{i}\right\| \geq \rho / 3 n$. By Lemma 4.5, $\left\|v_{i}-\tau_{k_{i} N} w_{i}\right\| \leq \rho / 15 n$ so that $\left\|u_{i}-\tau_{k_{i} N} w_{i}\right\| \geq 4 \rho / 15 n=4 \delta$ and $\left\|u_{i}-\tau_{k_{i} N} w_{i}\right\| \leq$ $\rho \leq C_{3}$. As a consequence, $u_{i} \in X \backslash G_{\delta} \cap \varphi^{-1}\left[0, d+\varepsilon_{0}\right.$ [, and Lemmas 4.2, 4.5 give

$$
\left\|\varphi^{\prime}(u)\right\| \geq\left\|\varphi^{\prime}\left(u_{i}\right)\right\|-4 \varepsilon / \delta \geq 4 \varepsilon / \delta .
$$

We can now prove the claim of Theorem 3.2.

MAIn THEOREM. There exists $u \in M B$ satisfying $\varphi^{\prime}(u)=0$.

Proof. Let $\bar{S}:=\mathbf{B}\left(\sum_{i} \tau_{k_{i} N} F, \rho / 3\right), \bar{\delta}:=\rho / 3, \bar{\varepsilon}:=(2 n+2) \varepsilon / 4 n$, and $\bar{d}:=n d+(2 n-1) \varepsilon / 4 n$. If the theorem is false, then

$$
\left\|\varphi^{\prime}(u)\right\|>0 \text { for all } u \in \bar{S}_{2 \bar{\delta}} .
$$

By Lemma 4.8, for all $u \in\left(\bar{S}_{\bar{\delta}} \backslash \bar{S}\right),\left\|\varphi^{\prime}(u)\right\| \geq 60 n \varepsilon / \rho \geq 8 \bar{\varepsilon} / \bar{\delta}$. As $\rho / 3+2 \bar{\delta}<C_{2}$, $\bar{S}_{2 \bar{\delta}}$ is contained in a finite union of balls of radius smaller than $C_{2}$ and thus there is no Palais-Smale sequence $\left(u_{n}\right)$ inside $\bar{S}_{2} \bar{\delta}$. Indeed otherwise, by Lemma 2.6, $\left(u_{n}\right)$ would converge to a critical point $u \in \bar{S}_{2 \bar{\delta}}$, contradicting (9). Let $\bar{\eta}$ be the deformation given by Lemma 4.9 and $\bar{\Psi}:=\bar{\eta}(1, \Psi)$. As $\Psi\left([0,1]^{n}\right) \subset \bar{S}$, $\bar{\Psi}\left([0,1]^{n}\right) \subset \bar{S}_{\bar{\delta}} \subset M B$, so that $\bar{\Psi} \in \Theta$. We get the contradiction to Lemma 4.7:

$$
\begin{aligned}
\bar{\Psi}\left([0,1]^{n}\right) & \left.\left.\left.\left.\subset \bar{\eta}\left(1, \varphi^{-1}\right]-\infty, \bar{d}+\bar{\varepsilon}\right]\right) \subset \varphi^{-1}(]-\infty, \bar{d}-\bar{\varepsilon}\right]\right) \\
& \left.\left.=\varphi^{-1}(]-\infty, n d-3 \varepsilon / 4 n\right]\right)
\end{aligned}
$$




\section{Appendix}

In this appendix, we establish a deformation lemma which has been used twice in the preceding section. In a "clasical" deformation lemma (see e.g. [10]) one asks a lower bound for $\left\|\varphi^{\prime}\right\|$ on a certain subset. This condiction has two consequences: on the one hand it garanties the existence of the flow for any positive time and on the other hand it implies a sufficient decrease of level along this flow. For this last consequence only, the requested condition is too strong and can be reduced (the second one will still be valid at the price of another condition introduced in [3] under the name $\overline{P S}$ ).

Lemma 4.9. Let $X$ be a Banach space, $\varphi \in \mathcal{C}^{1}(X, \mathbb{R}), S \subset X, d \in \mathbb{R}$, and $\varepsilon, \delta>0$ such that

(1) for all $u \in \varphi^{-1}[d-2 \varepsilon, d+2 \varepsilon] \cap S_{2 \delta}:\left\|\varphi^{\prime}\right\|>0$,

(2) for all $\left.u \in \varphi^{-1}[d-\varepsilon, d+\varepsilon] \cap S_{\delta} \backslash S\right):\left\|\varphi^{\prime}\right\|>8 \varepsilon / \delta$,

(3) for any Palais-Smale sequence $\left(u_{n}\right)$ for $\varphi$ at a level $b \in[d-2 \varepsilon, d+2 \varepsilon]$ and such that $\left\|u_{n+1}-u_{n}\right\| \rightarrow 0$ there exists $n_{0} \in \mathbb{N}: u_{n_{0}} \notin S_{2 \delta}$.

Then there exists $\eta \in \mathcal{C}([0,1] \times X, X)$ such that

(a) $\eta(t, u)=u$ if $t=0$ or if $u \notin \varphi^{-1}[d-2 \varepsilon, d+2 \varepsilon] \cap S_{2 \delta}$.

(b) $\eta\left(1, \varphi^{d+\varepsilon} \cap S\right) \subset \varphi^{d-\varepsilon}$.

(c) $\varphi(\eta(\cdot, u)$ is non increasing for every $u \in X$.

(d) $\eta(1, S) \subset S_{\delta}$.

Proof. The proof is identical to the one that can be found in [10] except for one point: the vector field is no longer bounded and we have to prove that the flow is well defined for any positive time.

If not, there exist $u \in X$ and $T>0$ such that $\eta(t, u)$ is defined only for $t \in[0, T[$. Let $l(t)$ be the length of the integral curve starting from $u$, we thus have $\lim _{t \rightarrow T} l(t)=\infty$. Define the 1 -speed equivalent of this curve

$$
\bar{\eta}(t):=\eta\left(l^{-1}(t), u\right),
$$

and observe that by change of variable formula,

$$
\begin{aligned}
\int_{0}^{\infty} \| \varphi^{\prime}(\bar{\eta}(t) \| d t & =\int_{0}^{\infty}\left\|\varphi^{\prime}\left(\eta\left(l^{-1}(t), u\right)\right)\right\| d t \\
& \leq \int_{0}^{T}\left\|\varphi^{\prime}(\eta(s, u))\right\| \cdot \sqrt{1+\|g(\eta(s, u))\|^{2}} d s \leq 2 T<\infty,
\end{aligned}
$$

where $g$ is the pseudo-gradient field associated to $\varphi$. As a consequence, for every $k \in \mathbb{N}$ there exists $R_{k}>0$ and a sequence $\left(t_{j}^{k}\right)_{1 \leq j}$ such that

(1) $t_{j}^{k} \in\left[R_{k}+j / k, R_{k}+(j+1) / k\right]$,

(2) $\left\|\varphi^{\prime}\left(\bar{\eta}\left(t_{j}^{k}\right)\right)\right\| \leq 1 / k \quad 1 \leq j$. 
(Without loss of generality we can assume that $R_{k}>R_{j}$ if $k>j$.)

Define

$$
\bar{t}_{j}^{k}:=t_{j}^{k} \quad \text { while } \quad t_{j}^{k}<R_{k+1},
$$

and let $\left(t_{j}\right)_{j \geq 1}$ be the sequence obtained by concatenation of the $\left(\bar{t}_{j}^{k}\right)$. By construction, $\left(\bar{\eta}\left(t_{j}\right)\right)_{j \geq 1}$ is a Palais-Smale sequence for $\varphi$ at a level $b \in[d-2 \varepsilon, d+2 \varepsilon]$ such that

$$
\left\|\bar{\eta}\left(t_{j+1}\right)-\bar{\eta}\left(t_{j}\right)\right\| \leq\left|t_{j+1}-t_{j}\right| \rightarrow 0 \quad(\bar{\eta} \text { is a } 1 \text {-speed curve }) .
$$

But then by condition (3), there exists $j_{0} \geq 1$ such that $\bar{\eta}\left(t_{0}\right) \notin S_{2 \delta}$. This is a contradiction because the vector field is zero outside $S_{2 \delta}$ and the integral curve would stay there for any positive time.

\section{REFERENCES}

[1] G. Arioli, F. Gazzola and S. Terracini, Multibump periodic motions of an infinite lattice of particles, Math. Z. 223 (1996), 627-642.

[2] P. Caldiroli and P. Montecchiari, Homoclinic orbits for second order Hamiltonian systems with potential changing sign, Comm. Appl. Nonlinear Anal. 2 (1994), 97-129.

[3] V. Coti Zelati, I. Ekeland and E. SÉRÉ, A variational approach to homoclinic orbits in Hamiltonian systems, Math. Ann. 288 (1990), 133-160.

[4] V. Coti Zelati and P. H. Rabinowitz, Homoclinic orbits for second order Hamiltonian systems possessing superquadratic potentials, J. Amer. Math. Soc. 4 (1991), 693-727.

[5] - Homoclinic type solutions for a semilinear elliptic PDE on $\mathbb{R}^{n}$, Comm. Pure Appl. Math. XLV (1992), 1217-1269.

[6] W. Hurewicz and H. Wallman, Dimension Theory, Princeton University Press, 1948.

[7] E. SÉRÉ, Existence of infinitely many homoclinic orbits in Hamiltonian systems, Math. Z. 209 (1992), 27-42.

[8] D. Smets, Ondes solitaires et méthodes variationnelles, Mémoire de licence, 96-97; Université Catholique de Louvain.

[9] D. Smets And M. Willem, Solitary waves with prescribed speed on infinite lattices, J. Funct. Anal. 149, 1 (1997), 266-275.

[10] M. Willem, Minimax Theorems, Birkhäuser, Basel, 1996.

Didier Smets

Université Catholique de Louvain

Département de Mathématique

Chemin du Cyclotron, 2

B1348 Louvain-la-Neuve, BELGIQUE

E-mail address: smets@amm.ucl.ac.be

TMNA : Volume $12-1998-\mathrm{N}^{\circ} 1$ 